\title{
Stability in distribution of neutral stochastic partial differential delay equations driven by $\alpha$-stable process
}

\author{
Yanchao Zang* and Junping Li
}

"Correspondence:
zycmail@126.com
School of Mathematics and
Statistics, Central South University,
Changsha, Hunan 410075, P.R. China

Changsha, Hunan 410075, P.R. China

\begin{abstract}
We consider a class of neutral stochastic partial differential equations driven by an $\alpha$-stable process. We prove the existence and uniqueness of the mild solution to the equation by the Banach fixed-point theorem under some suitable assumptions. Sufficient conditions for the stability in the distribution of the mild solution are derived.

MSC: $39 \mathrm{~A} 11 ; 37 \mathrm{H} 10$

Keywords: neutral stochastic partial differential equation; $\alpha$-stable process; mild solution; stability in distribution
\end{abstract}

\section{Introduction}

The theory of stochastic partial differential equations has been widely applied in scientific fields such as physics, mechanical engineering, and economics. Especially, the study of stochastic neutral functional differential equations has received a great deal of attention in recent years. For example, Bao et al. [1] extended the existence and uniqueness of mild solutions to a class of more general stochastic neutral partial functional differential equations under non-Lipschitz conditions. Caraballo et al. [2] investigated the exponential stability and ultimate boundedness of the solutions to a class of neutral stochastic semilinear partial delay differential equations.

Also the stability in a distribution is an important notion like the stability in probability or in the moment of stochastic differential equations. Such a stability is much weaker than stability in probability and it is useful sometimes to know whether or not the probability distribution of the solution will converge to some distribution but not necessarily to zero. There is an extensive literature concerned with the stability in the distribution of stochastic differential equations. Using an excellent stopping time technique and an $M$-matrix trick, Yuan and Mao [3] investigated the stability in the distribution of nonlinear SDEs with Markovian switching. Yuan et al. [4] discussed a class of stochastic differential delay equation with Markovian switching, where the sufficient conditions of stability in the distribution were established. Tan et al. [5] considered weak convergence of functional SDEs with variable delays. For the case of stochastic partial differential equations, we refer to Bao et al. $[1,6]$. Furthermore, for the nonlinear regime switching jump diffusion, we can refer to Yang and Yin [7].

O2014 Zang and Li; licensee Springer. This is an Open Access article distributed under the terms of the Creative Commons Attribution License (http://creativecommons.org/licenses/by/2.0), which permits unrestricted use, distribution, and reproduction in any medium, provided the original work is properly cited. 
Although many scholars have discussed the stability in the distribution of SDEs or functional SDEs where the noises are Brownian motion and jumps, the methods applied therein will not work if the considered noises are $\alpha$-stable processes. As we know, for an $\alpha$ stable process $(0<\alpha<2)$, it only has a $p<\alpha$ moment. Therefore, some useful techniques involved in the above references, such as the Burkholder-Davis-Gundy inequality and $\mathrm{Da}$ Prato-Kwapien-Zabczyk's factorization technique [8], are not available. On the other hand, it seems that little is known about the stability in the distribution of the neutral stochastic partial differential equations driven by an $\alpha$-stable process, and there are few systematic works so far in which the noise source is an $\alpha$-stable process as well. For more studies of stochastic systems driven by stable processes, we refer to [9-11].

In this paper, we study the existence, uniqueness, and stability in the distribution of mild solutions for the following neutral stochastic differential equation with finite delay:

$$
\left\{\begin{array}{l}
d[X(t)+g(X(t-\tau))]=[A X(t)+f(X(t-\tau))] d t+d Z(t), \quad 0 \leq t \leq T, \\
X_{0}(\cdot)=\xi \in D([-\tau, 0], H),
\end{array}\right.
$$

where $D([-\tau, 0], H)$ is the space of all càdlág functions paths from $[-\tau, 0]$ into $H$, a Hilbert space, equipped with the supremum norm $\|\varphi\|_{\infty}=\sup _{-\tau \leq t \leq 0}\|\varphi(t)\|_{H}$. And $g, f: H \rightarrow H$ are given functions to be specified later.

The contents of the paper are as follows. In Section 2, we briefly present some basic notations and preliminaries. In Section 3 the existence and uniqueness of mild solutions are proved. In the last section, we devote to give the sufficient conditions of the stability in the distribution of the mild solution to Eq. (1).

\section{Preliminary}

Let $\left(H,\langle\cdot, \cdot\rangle_{H},\|\cdot\|_{H}\right)$ be a real separable Hilbert space. Denote by $\mathcal{D}:=D([-\tau, 0] ; H)$ the space of all $H$-valued càdlág functions defined on $[-\tau, 0]$ equipped with the uniform norm $\|\xi\|_{\infty}:=\sup _{-\tau \leq \theta \leq 0}\|\xi(\theta)\|_{H}$. Recall that a path $f:[-\tau, 0] \mapsto H$ is called càdlág if it is rightcontinuous having finite left-hand limits.

Let $Z(t)$ be a cylindrical $\alpha$-stable process, $\alpha \in(0,2)$, defined by

$$
Z(t):=\sum_{m=1}^{\infty} \beta_{m} Z_{m}(t) e_{m}
$$

Here $\left\{e_{m}\right\}_{m \geq 1}$ is an orthonormal basis of $H,\left\{Z_{m}(t)\right\}_{m \geq 1}$ are independent, real-valued, normalized, symmetric $\alpha$-stable Lévy processes defined on stochastic basis $\left(\Omega, \mathcal{F},\left\{\mathcal{F}_{t}\right\}, P\right)$, and $\left\{\beta_{m}\right\}_{m \geq 1}$ is a sequence of positive numbers. Recall that a stochastic process $\left\{Z_{\alpha, \beta}(t)\right.$ : $t \geq 0\}$ is called an $\alpha$-stable Lévy process if

(i) $Z_{\alpha, \beta}(0)=0$ a.s.;

(ii) $Z_{\alpha, \beta}(t)$ has independent increments;

(iii) $Z_{\alpha, \beta}(t)-Z_{\alpha, \beta}(s) \sim \eta$ for any $0 \leq s<t<\infty$,

where $\eta$ stands for an $\alpha$-stable random variable, which is uniquely determined by its characteristic function involving four parameters: $\alpha \in(0,2]$, the index of stability; $\beta \in[-1,1]$, the skewness parameter; $\sigma \in(0, \infty)$, the scale parameter; $\mu \in(-\infty, \infty)$, the shift, and which has the form

$$
\phi_{\eta}(u)=E \exp (i u \eta)=\exp \left\{-\sigma^{\alpha}|u|^{\alpha}(1-i \beta \operatorname{sgn}(u) \Phi)+i \mu u\right\}, \quad u \in \mathbb{R},
$$


where $\Phi=\tan (\pi \alpha / 2)$ for $\alpha \neq 1$ and $\Phi=-(2 / \pi) \log |u|$ for $\alpha=1$. We call $\eta$ is strictly $\alpha$-stable whenever $\mu=0$, and if, in addition, $\beta=0, \eta$ is said to be symmetric $\alpha$-stable. For a realvalued normalized (standard) symmetric $\alpha$-stable Lévy process $z(t), \alpha \in(0,2)$, it has the characteristic function

$$
E \exp (i u z(t))=e^{-t|u|^{\alpha}}, \quad u \in \mathbb{R},
$$

and the Lévy measure $\lambda_{\alpha}(d x):=\frac{c_{\alpha}}{|x|+\alpha}, x \in \mathbb{R}-0$, where $c_{\alpha}$ is some constant. For more details of $\alpha$-stable processes, we can refer to [12] and [13].

Throughout the paper we impose the following assumptions:

(H1) The operator $(A, D(A))$ is a self-adjoint compact operator on the Hilbert space $H$ which is separable such that $-A$ has discrete spectrum $0<\lambda_{1} \leq \lambda_{2} \leq \cdots \leq \lambda_{m} \leq \cdots \leq \lim _{m \rightarrow \infty} \lambda_{m}=\infty$ with corresponding eigenbasis $\left\{e_{m}\right\}_{m \geq 1}$ of $H$. In this case $A$ generates a compact $C_{0}$-semigroup $S(t)=e^{t A}, t \geq 0$, such that $\left\|e^{t A}\right\| \leq e^{-\lambda_{1} t}$.

(H2) There exists a positive constant $K_{1}$ such that for all $x, y \in H$

$$
\|f(x)-f(y)\|_{H} \leq K_{1}\|x-y\|_{H}, \quad\|f(x)\|_{H} \leq K_{1}\left(1+\|x\|_{H}\right) .
$$

(H3) There exist $k \in(0,1)$ and a positive constant $K_{2}$ such that for all $x, y \in H$ and

(i) $\left\|(-A)^{k} g(x)-(-A)^{k} g(y)\right\|_{H} \leq K_{2}\|x-y\|_{H}$;

(ii) $\left\|(-A)^{k} g(x)\right\|_{H} \leq K_{2}\left(1+\|x\|_{H}\right)$;

(iii) The constants $K_{2}$ and $k$ satisfy $10 K_{2}^{p}\left\|(-A)^{-k}\right\|^{p}<1$.

(H4) There exists $\theta \in(0, k)$ such that $\alpha \theta \in(0,1)$ and $\delta:=\sum_{m=1}^{\infty} \frac{\beta_{m}^{\alpha}}{\lambda_{m}^{1-\alpha \theta}}<\infty$.

The following two lemmas will play an important role in proving our main results. So let us state them now.

Lemma 2.1 [14] Under (H1), for any $k \in(0,1]$ and $x \in D\left((-A)^{k}\right)$

$$
e^{t A}(-A)^{k} x=(-A)^{k} e^{t A} x
$$

and there exists $M_{k}>0$ such that for any $t>0$

$$
\left\|(-A)^{k} e^{t A}\right\| \leq M_{k} t^{-k} e^{-\lambda t}
$$

Lemma 2.2 [11] Let $T>0$ be arbitrary. For all $0 \leq \theta<\beta-\frac{1}{2 \alpha}$ and all $0<p<\alpha$, we have

$$
E \sup _{0 \leq t \leq T}\left\|(-A)^{\theta} Z_{A}(t)\right\|_{H}^{p} \leq C T^{\frac{p}{\alpha}}
$$

where $Z_{A}(t):=\int_{0}^{t} e^{(t-s) A} d Z(s), Z(s)$ is a cylindrical $\alpha$-stable process having the form of (2) and $C$ depends on $\alpha, \theta, \beta, p$.

Remark 2.1 In Lemma 2.2, the constant $\beta$ satisfies $\beta>\frac{1}{2}+\frac{1}{2 \alpha}$ so that the convolutions $Z_{A}(t)$ are in $H$. Moreover, there exist some $C_{1}, C_{2}>0$ such that $C_{1} \lambda_{k}^{-\beta} \leq\left|\beta_{k}\right| \leq C_{2} \lambda_{k}^{-\beta}$. 


\section{Existence and uniqueness}

The aim of this section is to establish the existence and uniqueness of the mild solution to Eq. (1). First, we give the following definition of mild solutions for Eq. (1).

Definition 3.1 An $\mathcal{F}_{t}$-adapted càdlág stochastic process $X(t), t \in[-\tau, T]$, is called a mild solution of Eq. (1) if it has the following properties:

(a) $X_{0}=\xi \in D([-\tau, 0], H)$.

(b) For arbitrary $t \in[0, T]$

$$
\begin{aligned}
X(t)= & e^{t A}(\xi(0)+g(\xi(-\tau)))-g(X(t-\tau)) \\
& -\int_{0}^{t} A e^{(t-s) A} g(X(s-\tau)) d s+\int_{0}^{t} e^{(t-s) A} f(X(s-\tau)) d s \\
& +\int_{0}^{t} e^{(t-s) A} d Z(s), \quad \text { a.s. }
\end{aligned}
$$

We shall denote by $S_{T}$ the Banach space of all of càdlág $H$-valued processes $X(t) \in$ $D([-\tau, T], H)$ with initial data $X(t)=\xi(t)$ for $t \in[-\tau, 0]$, and

$$
\|X(t)\|_{S_{T}}:=\left(E \sup _{-\tau \leq t \leq T}\|X(t)\|_{H}^{p}\right)^{\frac{1}{p}}<\infty .
$$

We have the following result.

Theorem 3.1 Suppose the assumptions (H1)-(H4) hold and let $p \in(1, \alpha), \alpha \in(1,2)$. Then, for any initial datum $\xi \in L^{p}(\Omega, D([-\tau, 0] ; H))$, there exists a unique mild solution $X(t)$ of Eq. (1) in $S_{T}$ and there exists a constant $C_{T}$, independent of $\xi$, such that

$$
E \sup _{t \in[0, T]}\|X(t)\|_{H}^{p} \leq C_{T}\left(1+E\|\xi\|_{\infty}^{p}\right) .
$$

Proof For arbitrary $\xi \in L^{p}(\Omega, D([-\tau, 0] ; H))$ and $X \in S_{T}$, define an operator $\Lambda$ on $S_{T}$ by that $\Lambda(X)(t)=\xi(t), t \in[-\tau, 0]$, and

$$
\begin{aligned}
\Lambda(X)(t)= & e^{t A}(\xi(0)+g(\xi(-\tau)))-g(X(t-\tau))-\int_{0}^{t} A e^{(t-s) A} g(X(s-\tau)) d s \\
& +\int_{0}^{t} e^{(t-s) A} f(X(s-\tau)) d s+\int_{0}^{t} e^{(t-s) A} d Z(s), \quad t \in[0, T] .
\end{aligned}
$$

The required assertion follows if we show that the operator $\Lambda$ has a fixed point in the space $S_{T}$ by the Banach fixed-point theorem. We divide the proof into two steps.

Step 1. We show that $\Lambda(X)(t) \subset S_{T}$ for $t \in[-\tau, T]$. It is trivial for the case $t \in[-\tau, 0]$. For $0 \leq t \leq T$, and for any fixed $X \in S_{T}$, using the trivial inequality: $\left(\sum_{i=1}^{n} a_{i}\right)^{p} \leq C_{r}\left(\sum_{i=1}^{n} a_{i}^{p}\right)$, here $C_{r}=1$ when $p \leq 1, C_{r}=n^{p-1}$ when $p>1$, we have

$$
\begin{aligned}
E \sup _{0 \leq t \leq T}\|\Lambda(X)(t)\|_{H}^{p} \leq & 5^{p-1} E \sup _{0 \leq t \leq T}\left\|e^{t A}(\xi(0)+g(\xi(-\tau)))\right\|_{H}^{p} \\
& +5^{p-1} E \sup _{0 \leq t \leq T}\|g(X(t-\tau))\|_{H}^{p}
\end{aligned}
$$




$$
\begin{aligned}
& \quad+5^{p-1} E \sup _{0 \leq t \leq T}\left\|\int_{0}^{t} A e^{(t-s) A} g(X(s-\tau)) d s\right\|_{H}^{p} \\
& +5^{p-1} E \sup _{0 \leq t \leq T}\left\|\int_{0}^{t} e^{(t-s) A} f(X(s-\tau)) d s\right\|_{H}^{p} \\
& \quad+5^{p-1} E \sup _{0 \leq t \leq T}\left\|\int_{0}^{t} e^{(t-s) A} d Z(s)\right\|_{H}^{p} \\
& =: \sum_{i=1}^{5} I_{i} .
\end{aligned}
$$

From (H1), (H3), and the boundedness of $(-A)^{-k}$ for $k \in(0,1)$, we get

$$
\begin{aligned}
I_{1} & =5^{p-1} E \sup _{0 \leq t \leq T}\left\|(-A)^{-k} e^{t A}(-A)^{k}(\xi(0)+g(\xi(-\tau)))\right\|_{H}^{p} \\
& \leq 10^{p-1} E\|\xi\|_{\infty}^{p}+10^{p-1}\left\|(-A)^{-k}\right\|^{p} K_{2}^{p} E\left(1+\|\xi\|_{\infty}\right)^{p} \\
& \leq M_{k, p}\left(1+E\|\xi\|_{\infty}^{p}\right),
\end{aligned}
$$

where $M_{k, p}$ is a constant only depending on $k$ and $p$. For the second term, $I_{2}$, using the assumption (H3) again, we have

$$
\begin{aligned}
I_{2} & \leq 5^{p-1}\left\|(-A)^{-k}\right\|^{p} E \sup _{0 \leq t \leq T}\left\|(-A)^{k} g(X(t-\tau))\right\|_{H}^{p} \\
& \leq 5^{p-1}\left\|(-A)^{-k}\right\|^{p} K_{2}^{p} E \sup _{0 \leq t \leq T}\left(1+\|X(t-\tau)\|_{H}\right)^{p} \\
& \leq 10^{p-1}\left\|(-A)^{-k}\right\|^{p} K_{2}^{p}\left(1+E \sup _{-\tau \leq t \leq T}\|X(t)\|_{H}^{p}\right) .
\end{aligned}
$$

We consider the third term, $I_{3}$ : apply Lemma 2.1, use the assumption (H3) and Hölder's inequality, and we derive

$$
\begin{aligned}
I_{3} & =5^{p-1} E \sup _{0 \leq t \leq T}\left\|\int_{0}^{t}(-A)^{1-k} e^{(t-s) A}(-A)^{k} g(X(s-\tau)) d s\right\|_{H}^{p} \\
& \leq 5^{p-1} M_{1-k}^{p} K_{2}^{p} E \sup _{0 \leq t \leq T}\left(\int_{0}^{t}(t-s)^{-(1-k)} e^{-\lambda(t-s)}\left(1+\|X(s-\tau)\|_{H}\right) d s\right)^{p} \\
& \leq 10^{p-1} T M_{1-k}^{p} K_{2}^{p} \lambda^{(1-p k)}\left|\Gamma\left(\frac{p k-1}{p-1}\right)\right|^{p-1}\left(1+E \sup _{-\tau \leq t \leq T}\|X(t)\|_{H}^{p}\right) .
\end{aligned}
$$

Similarly, for the fourth term, $I_{4}$, using Hölder's inequality, we obtain

$$
\begin{aligned}
I_{4} & \leq 5^{p-1} K_{1}^{p} E \sup _{0 \leq t \leq T}\left(\int_{0}^{t} e^{-\lambda(t-s)}\left(1+\|X(s-\tau)\|_{H}\right) d s\right)^{p} \\
& \leq 5^{p-1} K_{1}^{p} \sup _{0 \leq t \leq T}\left(\int_{0}^{t} e^{-\lambda(t-s)} d s\right)^{p-1} E \sup _{0 \leq t \leq T} \int_{0}^{t}\left(1+\|X(s-\tau)\|_{H}\right)^{p} d s \\
& \leq 10^{p-1} T K_{1}^{p} \lambda^{(1-p)}\left(1+E \sup _{-\tau \leq t \leq T}\|X(t)\|_{H}^{p}\right) .
\end{aligned}
$$


Noting that $I_{5} \leq 5^{p-1}\left\|(-A)^{-\theta}\right\|^{p} E \sup _{0 \leq t \leq T}\left\|(-A)^{\theta} Z_{A}(t)\right\|_{H}^{p}$, and by Lemma 2.2, we immediately get

$$
I_{5} \leq 5^{p-1}\left\|(-A)^{-\theta}\right\|^{p} C T^{\frac{p}{\alpha}} .
$$

Thus, we derive from (5) to (10) that, for some constants $c_{1}, c_{2}, c_{3}$,

$$
E \sup _{0 \leq t \leq T}\|\Lambda(X)(t)\|_{H}^{p} \leq c_{1}+c_{2} E\|\xi\|_{\infty}^{p}+c_{3} E \sup _{0 \leq t \leq T}\|X(t)\|_{H}^{p}
$$

Hence, $\Lambda(X)(t) \subset S_{T}$.

Step 2. We shall show that the mapping $\Lambda$ is contractive. Let $X, Y \in S_{T}$. For any fixed $t \in[0, T]$, we have

$$
\begin{aligned}
E \sup _{0 \leq t \leq T} & \|\Lambda(X)(t)-\Lambda(Y)(t)\|_{H}^{p} \\
\leq & 3^{p-1} E \sup _{0 \leq t \leq T}\|g(X(t-\tau))-g(Y(t-\tau))\|_{H}^{p} \\
& +3^{p-1} E \sup _{0 \leq t \leq T}\left\|\int_{0}^{t} A e^{(t-s) A}[g(X(s-\tau))-g(Y(s-\tau))] d s\right\|_{H}^{p} \\
& +3^{p-1} E \sup _{0 \leq t \leq T}\left\|\int_{0}^{t} e^{(t-s) A}[f(X(s-\tau))-f(Y(s-\tau))] d s\right\|_{H}^{p} .
\end{aligned}
$$

By the assumptions (H2), (H3), and Hölder's inequality, we obtain

$$
\begin{aligned}
& E \sup _{0 \leq t \leq T}\|\Lambda(X)(t)-\Lambda(Y)(t)\|_{H}^{p} \\
& \leq 3^{p-1} K_{2}^{p}\left\|(-A)^{-k}\right\|^{p} E \sup _{0 \leq t \leq T}\|X(t)-Y(t)\|_{H}^{p} \\
&+3^{p-1} M_{1-k}^{p} K_{2}^{p} T \lambda^{(1-p k)}\left|\Gamma\left(\frac{p k-1}{p-1}\right)\right|^{p-1} E \sup _{0 \leq t \leq T}\|X(t)-Y(t)\|_{H}^{p} \\
&+3^{p-1} K_{1}^{p} T \lambda^{(1-p)} E \sup _{0 \leq t \leq T}\|X(t)-Y(t)\|_{H}^{p} \\
& \leq 3^{p-1}\left(K_{2}^{p}\left\|(-A)^{-k}\right\|^{p}+C T\right) E \sup _{0 \leq t \leq T}\|X(t)-Y(t)\|_{H}^{p},
\end{aligned}
$$

where $X(t)=Y(t)$ on $[-\tau, 0]$, and $C>0$ is a bounded constant. Hence, by the condition $10 K_{2}^{p}\left\|(-A)^{-k}\right\|^{p}<1$, choosing sufficiently small $T_{1}$ such that $3^{p-1}\left(K_{2}^{p}\left\|(-A)^{-k}\right\|^{p}+C T_{1}\right)<1$, we can conclude that $\Lambda$ is contractive. Therefore, by the contraction principle, we have a mild solution of Eq. (1) on [0, $\left.T_{1}\right]$. Moreover with such a $T_{1}$ we get from (11) for the solution of Eq. (1)

$$
E \sup _{0 \leq t \leq T_{1}}\|X(t)\|_{H}^{p} \leq \frac{1}{1-c_{3}}\left[c_{1}+c_{2} E\|\xi\|_{\infty}^{p}\right]
$$

which is inequality (4). The solution can be extended to the entire interval $[0, T]$ in finite steps by repeating the above procedure on intervals $\left[0, T_{1}\right],\left[T_{1}, 2 T_{1}\right], \ldots$ with $T_{1}$ such that $c_{3}\left(T_{1}\right)<1$. This completes the proof. 


\section{Asymptotic stability in the distribution}

In this section, we shall derive some sufficient conditions on the stability in the distribution for the process $Y(t)=X_{t}$ on $t \geq 0$. We need to introduce some more notations. For $t \geq 0$, let $X(t ; \xi)$ be the solution of Eq. (1) with initial datum $X_{0}=\xi \in D([-\tau, 0], H)$. Correspondingly, $X_{t}(\xi)=\{X(t+\theta ; \xi):-\tau \leq \theta \leq 0\}$ on $t \geq 0$. As usual, $\left\{X_{t}\right\}_{t \geq 0}$ is called the segment process of $\{X(t)\}_{t \geq-\tau}$. Denote by $p(\xi, t, d \zeta)$ the transition probability of the process $Y(t)=X_{t}(\xi)$, then $Y(t)$ is a time homogeneous Markov process according to Mohammed [15].

Now, we introduce the concept of stability in the distribution and prepare some useful lemmas as follows.

Definition 4.1 [16] The process $Y(t)$ is said to be asymptotically stable in the distribution if there exists a probability measure $\pi(\cdot)$ on $D([-\tau, 0] ; H)$ such that the transition probability function $p(\xi, t, d \zeta)$ of $Y(t)$ converges weakly to $\pi(d \zeta)$ as $t \rightarrow \infty$ for every $\xi \in D([-\tau, 0] ; H)$. Equation (1) is said to be asymptotically stable in the distribution if the solution process $Y(t)=X_{t}(\xi)$ is asymptotically stable in the distribution.

By Yuan et al. [4], to show the stability in the distribution of Eq. (1), it is sufficient to verify that for some $p>0$ and bounded subset $U \subset \mathcal{D}$,

(N1) $\sup _{t \geq 0} \sup _{\xi \in U} E\left\|X_{t}(\xi)\right\|_{\infty}^{p}<\infty$;

(N2) $\lim _{t \rightarrow \infty} \sup _{\xi, \eta \in U} E\left\|X_{t}(\xi)-X_{t}(\eta)\right\|_{\infty}^{p}=0$, for every $x, y, z \in X$.

In what follows, we need to prepare some useful lemmas to demonstrate that (N1) and (N2) hold under some imposed conditions.

Lemma 4.1 Let $y:[-\tau,+\infty) \rightarrow[0,+\infty)$ be Borel measurable. If $y(t)$ is a solution of the delay integral inequality, then

$$
y(t) \leq \begin{cases}\|\phi\|_{\infty} e^{-r t}+b_{1}\left\|y_{t}\right\|_{\infty}+b_{2} \int_{0}^{t} e^{-r(t-s)}\left\|y_{s}\right\|_{\infty} d s+J, & t \geq 0, \\ \phi(t), & t \in[-\tau, 0],\end{cases}
$$

where $\phi(t) \in D([-\tau, 0],[0,+\infty)), r>0, b_{1}, b_{2}$, and J are nonnegative constants. If $\|\phi\|_{\infty} \leq K$ for some constant $K>0$ and

$$
b_{1}+\frac{b_{2}}{r}:=\rho<1,
$$

then there are constants $\gamma \in(0, r)$ and $N \geq K$ such that

$$
y(t) \leq N e^{-\gamma t}+\frac{J}{1-\rho}, \quad \forall t \geq 0,
$$

where $\gamma$ and $N$ satisfy

$$
\rho_{\gamma}:=b_{1} e^{\gamma \tau}+\frac{b_{2} e^{\gamma \tau}}{r-\gamma}<1 \quad \text { and } \quad N \geq \frac{K}{1-\rho_{\gamma}},
$$

or $b_{2} \neq 0$ and

$$
\rho_{\gamma} \leq 1 \quad \text { and } \quad N \geq \frac{(r-\gamma)\left[K-\frac{b_{2} J}{r(1-\rho)}\right]}{b_{2} e^{\gamma \tau}} .
$$


Proof The proof is similar to Lemma 3.1 of [17], so we omit it here.

Lemma 4.2 Assume that (H1)-(H4) hold, and that the inequality

$$
\rho:=10\left(\left\|(-A)^{-k}\right\|^{p} K_{2}^{p}+M_{1-k}^{p} K_{2}^{p} \lambda^{-p k}\left|\Gamma\left(\frac{p k-1}{p-1}\right)\right|^{p-1}+\lambda^{-p} K_{1}^{p}\right)<1
$$

holds for $k \in(0,1], p \in(1, \alpha)$, where $\Gamma(\cdot)$ is the gamma function. Then

$$
\sup _{0 \leq t<\infty} E\left\|X_{t}(\xi)\right\|_{\infty}^{p}<\infty
$$

for any $\xi \in D([-\tau, 0], H)$.

Proof First, we shall show $\sup _{-\tau \leq t<\infty} E\|X(t)\|_{H}^{p}<\infty$. For $p \in(1, \alpha)$, we obtain from (3) that

$$
\begin{aligned}
E\|X(t)\|_{H}^{p} \leq & 5 E\left\|e^{t A}[\xi(0)+g(\xi(-\tau))]\right\|_{H}^{p} \\
& +5 E\|g(X(t-\tau))\|_{H}^{p}+5 E\left\|\int_{0}^{t} A e^{(t-s) A} g(X(s-\tau)) d s\right\|_{H}^{p} \\
& +5 E\left\|\int_{0}^{t} e^{(t-s) A} f(X(s-\tau)) d s\right\|_{H}^{p}+5 E\left\|\int_{0}^{t} e^{(t-s) A} d Z(s)\right\|_{H}^{p} \\
= & : \sum_{i=1}^{5} J_{i}
\end{aligned}
$$

where $J_{i}(i=1,2, \ldots, 5)$ stands for the $i$ th term behind the first inequality. It follows from Lemma 2.1 and the assumption (H3) that

$$
\begin{aligned}
J_{1} & =5 E\left\|e^{t A}[\xi(0)+g(\xi(-\tau))]\right\|_{H}^{p} \\
& \leq 5 e^{-\lambda p t} E\left[\|\xi\|_{\infty}+\left\|(-A)^{-k}\right\| \cdot\left\|(-A)^{k} g(\xi(-\tau))\right\|_{H}\right]^{p} \\
& \leq 5 e^{-\lambda p t} E\left[\|\xi\|_{\infty}+K_{2}\left\|(-A)^{-k}\right\|\left(1+\|\xi\|_{\infty}\right)\right]^{p} \\
& \leq M^{*} e^{-\lambda p t}\left(1+E\|\xi\|_{\infty}^{p}\right),
\end{aligned}
$$

where $M^{*} \geq 1$ is an appropriate constant only depend on $k$ and $p$. For the second term $J_{2}$,

$$
\begin{aligned}
J_{2} & =5 E\|g(X(t-\tau))\|_{H}^{p} \leq 5 E\left[\left\|(-A)^{-k}\right\| \cdot\left\|(-A)^{k} g(X(t-\tau))\right\|_{H}\right]^{p} \\
& \leq 5\left\|(-A)^{-k}\right\|^{p} K_{2}^{p} E\left(1+\|X(t-\tau)\|_{H}\right)^{p} \\
& \leq 10\left\|(-A)^{-k}\right\|^{p} K_{2}^{p}\left(1+\sup _{-\tau \leq \theta \leq 0} E\|X(t+\theta)\|_{H}^{p}\right) .
\end{aligned}
$$

Similarly, by using Lemma 2.1, (H3), and Hölder's inequality, we obtain

$$
\begin{aligned}
J_{3} & =5 E\left\|\int_{0}^{t} A e^{(t-s) A} g(X(s-\tau)) d s\right\|_{H}^{p} \\
& \leq 5 E\left(\int_{0}^{t}\left\|(-A)^{1-k} e^{(t-s) A}(-A)^{k} g(X(s-\tau))\right\|_{H} d s\right)^{p}
\end{aligned}
$$


By (H2) and Hölder's inequality, we obtain

$$
\begin{aligned}
J_{4} & =5 E\left\|\int_{0}^{t} e^{(t-s) A} f(X(s-\tau)) d s\right\|_{H}^{p} \\
& \leq 5 E\left(\int_{0}^{t} e^{-\lambda(t-s)} K_{1}\left(1+\|X(s-\tau)\|_{H}\right) d s\right)^{p} \\
& \leq 10 \lambda^{1-p} K_{1}^{p} \int_{0}^{t} e^{-\lambda(t-s)}\left(1+\sup _{-\tau \leq \theta \leq 0} E\|X(s+\theta)\|_{H}^{p}\right) d s .
\end{aligned}
$$

For the estimation of $J_{5}$, by the argument of [10, Theorem 4.4] and applying (H4), we have

$$
J_{5}=5 E\left\|\int_{0}^{t} e^{(t-s) A} d Z(s)\right\|_{H}^{p} \leq 5 \tilde{c}_{p}\left(\sum_{m \geq 1} \beta_{m}^{\alpha} \frac{1-e^{-\alpha \lambda_{m} t}}{\alpha \lambda_{m}}\right)^{p / \alpha} \leq 5 \tilde{c}_{p}\left(\delta /\left(\alpha \lambda_{1}^{\alpha \theta}\right)\right)^{p / \alpha},
$$

where $\tilde{c}_{p}$ depends only on $p$. Thus, substituting (15)-(19) into (14), we get

$$
\begin{aligned}
E\|X(t)\|_{H}^{p} \leq & M^{*} e^{-\lambda p t} E\|\xi\|_{\infty}^{p}+b_{1} \sup _{-\tau \leq \theta \leq 0} E\|X(t+\theta)\|_{H}^{p} \\
& +b_{2} \int_{0}^{t} e^{-\lambda(t-s)} \sup _{-\tau \leq \theta \leq 0} E\|X(s+\theta)\|_{H}^{p} d s+J
\end{aligned}
$$

where $b_{1}=10\left\|(-A)^{-k}\right\|^{p} K_{2}^{p}, b_{2}=10\left(M_{1-k}^{p} K_{2}^{p} \lambda^{1-p k}\left|\Gamma\left(\frac{p k-1}{p-1}\right)\right|^{p-1}+\lambda^{1-p} K_{1}^{p}\right)$, and

$$
\begin{aligned}
J= & M^{*} e^{-\lambda p t}+10\left\|(-A)^{-k}\right\|^{p} K_{2}^{p}+10 M_{1-k}^{p} K_{2}^{p} \lambda^{-p k}\left|\Gamma\left(\frac{p k-1}{p-1}\right)\right|^{p-1} \\
& +10 \lambda^{-p} K_{1}^{p}+5 \tilde{c}_{p}\left(\delta /\left(\alpha \lambda_{1}^{\alpha \theta}\right)\right)^{p / \alpha} .
\end{aligned}
$$

In the light of (12), $\rho=b_{1}+\frac{b_{2}}{\lambda}<1$ holds. Furthermore, there exist $\bar{K} \geq 0, \bar{N}>0, \gamma \in(0, \lambda)$, such that

$$
M^{*} E\|\xi\|_{\infty}^{p} \leq \bar{K}, \quad \rho_{\gamma}=b_{1} e^{\gamma \tau}+\frac{b_{2} e^{\gamma \tau}}{\lambda-\gamma} \leq 1 \quad \text { and } \quad \frac{(\lambda-\gamma)\left[\bar{K}-\frac{b_{2} J}{\lambda(1-\bar{\rho})}\right]}{b_{2} e^{\gamma \tau}} \leq \bar{N}
$$

Consequently, combining (12), (20) with Lemma 4.1, we arrive at

$$
\sup _{-\tau \leq t<\infty} E\|X(t)\|_{H}^{p} \leq \bar{N} e^{\gamma \tau}+\frac{J}{(1-\rho)}<\infty
$$

Next, we use the following trivial inequality: for any $a, b, c, d, e \geq 0$ and $\epsilon>0$,

$$
(a+b+c+d+e)^{2} \leq(1+\epsilon) a^{2}+4\left(1+\frac{1}{\epsilon}\right)\left(b^{2}+c^{2}+d^{2}+e^{2}\right) .
$$


For any integer $n \geq 1$, similar to the above computations, one has

$$
\begin{aligned}
E\left\|X_{n \tau}(\xi)\right\|_{\infty}^{p} \leq & (1+\epsilon)^{\frac{p}{2}} E\left\|g\left(X_{(n-1) \tau}(\xi)\right)\right\|_{\infty}^{p} \\
& +2^{p}\left(1+\frac{1}{\epsilon}\right)^{\frac{p}{2}}\left\{E \sup _{-\tau \leq \theta \leq 0}\left\|e^{(\theta+\tau) A}[X((n-1) \tau)+g(X((n-2) \tau))]\right\|_{H}^{p}\right. \\
& +E \sup _{-\tau \leq \theta \leq 0}\left\|\int_{(n-1) \tau}^{n \tau+\theta} A e^{(n \tau+\theta-s) A} g(X(s-\tau)) d s\right\|_{H}^{p} \\
& +E \sup _{-\tau \leq \theta \leq 0}\left\|\int_{(n-1) \tau}^{n \tau+\theta} e^{(n \tau+\theta-s) A} f(X(s-\tau)) d s\right\|_{H}^{p} \\
& \left.+E \sup _{-\tau \leq \theta \leq 0}\left\|\int_{(n-1) \tau}^{n \tau+\theta} e^{(n \tau+\theta-s) A} d Z(s)\right\|_{H}^{p}\right\} \\
\leq & (1+\epsilon)^{p} K_{2}^{p}\left\|(-A)^{-k}\right\|^{p} E\|X(n-1) \tau(\xi)\|_{\infty}^{p} \\
& +C\left(1+\sup _{(n-2) \tau \leq t \leq(n-1) \tau} E\|X(t, \xi)\|_{H}^{p}+E \sup _{0 \leq t \leq \tau}\left\|\int_{0}^{t} e^{(t-s) A} d \tilde{Z}(s)\right\|_{H}^{p}\right),
\end{aligned}
$$

where $\tilde{Z}(s):=Z(s+(n-1) \tau)-Z((n-1) \tau)$ is also a cylindrical stable process, $C$ is a constant not depending on $n$ and its value is not important and may change from one line to another. Hence, according to (21) and Lemma 2.2,

$$
E\left\|X_{n \tau}(\xi)\right\|_{\infty}^{p} \leq(1+\epsilon)^{p} K_{2}^{p}\left\|(-A)^{-k}\right\|^{p} E\left\|X_{(n-1) \tau}(\xi)\right\|_{\infty}^{p}+C
$$

holds. In view of (iii) of (H3) and (21), there exist constants $\vartheta$ and $M$ such that, for sufficient small $\epsilon,(1+\epsilon)^{p} K_{2}^{p}\left\|(-A)^{-k}\right\|^{p}<\vartheta<1$, and for any integer $n \geq 1$,

$$
\begin{aligned}
E\left\|X_{n \tau}(\xi)\right\|_{\infty}^{p} & \leq \vartheta E\left\|X_{(n-1) \tau}(\xi)\right\|_{\infty}^{p}+M \\
& \leq \vartheta^{n}\|\xi\|_{\infty}^{p}+M\left\{1+\vartheta+\cdots+\vartheta^{n-1}\right\} \\
& \leq\|\xi\|_{\infty}^{p}+\frac{M}{1-\vartheta} .
\end{aligned}
$$

Observe that for any $t \geq 0$, there exists an $n \geq 0$ such that $t \in[n \tau,(n+1) \tau)$ and

$$
E\left\|X_{t}(\xi)\right\|_{\infty}^{p} \leq E\left\|X_{(n+1) \tau}(\xi)\right\|_{\infty}^{p}+E\left\|X_{n \tau}(\xi)\right\|_{\infty}^{p}
$$

Then the desired assertion (13) follows immediately from (22).

Lemma 4.3 Let the conditions of $(\mathrm{H} 1)-(\mathrm{H} 4)$ and (12) hold. Then for any bounded subset $K$ of $D([-\tau, 0], H)$,

$$
\lim _{t \rightarrow \infty} E\left\|X_{t}(\xi)-X_{t}(\eta)\right\|_{\infty}^{p}=0
$$

uniformly in $\xi, \eta \in K$.

Proof Since the argument is similar to Lemma 4.2, we only sketch the main proof to point out the difference with that of Lemma 4.2 here. First, we shall prove that

$$
\lim _{t \rightarrow \infty} E\|X(t, \xi)-X(t, \eta)\|_{H}^{p}=0
$$


uniformly in $\xi, \eta \in K$. Following a similar argument to derive (20), we can get

$$
\begin{aligned}
E\|X(t, \xi)-X(t, \eta)\|_{H}^{p}= & 4 E\left\|e^{t A}[\xi(0)+g(\xi(-\tau))-\eta(0)-g(\eta(-\tau))]\right\|_{H}^{p} \\
& +4 E\|g(X(t-\tau, \xi))-g(X(t-\tau, \eta))\|_{H}^{p} \\
& +4 E\left\|\int_{0}^{t} A e^{(t-s) A}[g(X(s-\tau, \xi))-g(X(s-\tau, \eta))] d s\right\|_{H}^{p} \\
& +4 E\left\|\int_{0}^{t} e^{(t-s) A}[f(X(s-\tau, \xi))-f(X(s-\tau, \eta))] d s\right\|_{H}^{p} \\
\leq & \tilde{b}_{1} e^{-\lambda p t}+\tilde{b}_{2} \sup _{-\tau \leq \theta \leq 0} E\|X(t+\theta, \xi)-X(t+\theta, \eta)\|_{H}^{p} \\
& +\tilde{b}_{3} \int_{0}^{t} e^{-\lambda(t-s)} \sup _{-\tau \leq \theta \leq 0} E\|X(s+\theta, \xi)-X(s+\theta, \eta)\|_{H}^{p} d s,
\end{aligned}
$$

where $\tilde{b}_{1}=8 E\|\xi-\eta\|_{\infty}^{p}\left(1+K_{2}^{p}\left\|(-A)^{-k}\right\|^{p}\right), \tilde{b}_{2}=4 K_{2}^{p}\left\|(-A)^{-k}\right\|^{p}$ and

$$
\tilde{b}_{3}=4\left(M_{1-k}^{p} K_{2}^{p} \lambda^{(1-p k)}\left|\Gamma\left(\frac{p k-1}{p-1}\right)\right|^{p-1}+\lambda^{1-p} K_{1}^{p}\right) .
$$

As a result, noting the condition (12) and using [18, Lemma 3.1], we derive from (24) that

$$
E\|X(t, \xi)-X(t, \eta)\|_{H}^{p} \leq M_{2} e^{-\mu t}, \quad t \geq-\tau
$$

where $\mu$ is a positive root of the equation $\left(\tilde{b}_{2}+\left(\tilde{b}_{3} / \lambda-\mu\right)\right)=1$ and $M_{2}=\max \left\{\tilde{b}_{1}(\lambda-\right.$ $\left.\mu) / \tilde{b}_{3} e^{\mu \tau}, \tilde{b}_{1}\right\}$. Hence,

$$
\lim _{t \rightarrow \infty} E\|X(t, \xi)-X(t, \eta)\|_{H}^{p}=0
$$

Now, for $t \geq 2 \tau$ and $-\tau \leq \theta \leq 0$, according to the fundamental inequality and assumptions (H1)-(H4), we arrive at

$$
\begin{aligned}
& E\left\|X_{t}(\xi)-X_{t}(\eta)+g\left(X_{t-\tau}(\xi)\right)-g\left(X_{t-\tau}(\eta)\right)\right\|_{\infty}^{p} \\
& \leq 3 E \sup _{-\tau \leq \theta \leq 0}\left\|e^{(\theta+\tau) A}[X(t-\tau, \xi)-X(t-\tau, \eta)+g(X(t-2 \tau, \xi))-g(X(t-2 \tau, \eta))]\right\|_{H}^{p} \\
& \quad+3 E \sup _{-\tau \leq \theta \leq 0}\left\|\int_{t-\tau}^{t+\theta} A e^{(t+\theta-s) A}[g(X(s-\tau, \xi))-g(X(s-\tau, \eta))] d s\right\|_{H}^{p} \\
& \quad+3 E \sup _{-\tau \leq \theta \leq 0}\left\|\int_{t-\tau}^{t+\theta} e^{(t+\theta-s) A}[f(X(s-\tau, \xi))-f(X(s-\tau, \eta))] d s\right\|_{H}^{p} \\
& \leq 6 E\|X(t-\tau, \xi)-X(t-\tau, \eta)\|_{H}^{p}+6 K_{2}^{p}\left\|(-A)^{-k}\right\|^{p} E\|X(t-2 \tau, \xi)-X(t-2 \tau, \eta)\|_{H}^{p} \\
& \quad+3 M_{1-k}^{p} K_{2}^{p} e^{\lambda p \tau} \lambda^{1-p k}\left|\Gamma\left(\frac{p k-1}{p-1}\right)\right|^{p-1} \int_{t-\tau}^{t} e^{-\lambda(t-s)} E\|X(s-\tau, \xi)-X(s-\tau, \eta)\|_{H}^{p} d s \\
& \quad+3 K_{1}^{p} e^{\lambda p \tau} \lambda^{1-p} \int_{t-\tau}^{t} e^{-\lambda(t-s)} E\|X(s-\tau, \xi)-X(s-\tau, \eta)\|_{H}^{p} d s .
\end{aligned}
$$


This, together with (25), yields

$$
\lim _{t \rightarrow \infty} E\left\|X_{t}(\xi)-X_{t}(\eta)+g\left(X_{t-\tau}(\xi)\right)-g\left(X_{t-\tau}(\eta)\right)\right\|_{\infty}^{p}=0 .
$$

Finally, we claim that the desired equation (23) must hold. If Eq. (23) is not true, then there exist positive constants $L$ and $T$, where, when $t \geq T$,

$$
E\left\|X_{t}(\xi)-X_{t}(\eta)\right\|_{\infty}^{p} \geq L
$$

On the other hand, when $t \rightarrow \infty$,

$$
\begin{aligned}
E^{\frac{1}{p}} & \left\|X_{t}(\xi)-X_{t}(\eta)+g\left(X_{t-\tau}(\xi)\right)-g\left(X_{t-\tau}(\eta)\right)\right\|_{\infty}^{p} \\
& \geq E^{\frac{1}{p}}\left\|X_{t}(\xi)-X_{t}(\eta)\right\|_{\infty}^{p}-K_{2}\left\|(-A)^{-k}\right\| E^{\frac{1}{p}}\left\|X_{t-\tau}(\xi)-X_{t-\tau}(\eta)\right\|_{\infty}^{p} \\
& \geq\left(1-K_{2}\left\|(-A)^{-k}\right\|\right) L .
\end{aligned}
$$

This obviously contradicts (27). Therefore, (23) holds.

In what follows we aim to prove the stability in the distribution of Eq. (1). Denote by $\mathcal{P}(D([-\tau, 0] ; H))$ the space of all probability measures on $D([-\tau, 0] ; H)$. For $P_{1}, P_{2} \in$ $\mathcal{P}(D([-\tau, 0] ; H))$, define

$$
d_{\mathbb{L}}\left(P_{1}, P_{2}\right)=\sup _{f \in \mathbb{L}}\left|\int_{H} f(\xi) P_{1}(d \xi)-\int_{H} f(\eta) P_{2}(d \eta)\right|,
$$

where, for any $\xi, \eta \in D([-\tau, 0] ; H)$,

$$
\mathbb{L}=\{f: D([-\tau, 0] ; H) \rightarrow R:|f(\xi)-f(\eta)| \leq\|\xi-\eta\| \text { and }|f(\cdot)| \leq 1\}
$$

Lemma 4.4 Let assumptions (H1)-(H4), (13), and (23) hold. Then, for any initial data $\xi \in$ $D([-\tau, 0] ; H),\{p(\xi, t, \cdot): t \geq 0\}$ is Cauchy in the space $\mathcal{P}(D([-\tau, 0], H))$ with the metric $d_{\mathbb{L}}$.

Proof For any fixed $\xi \in D([-\tau, 0] ; H)$, we need to show that for any $\epsilon>0$, there is a $T_{1}>0$ such that

$$
d_{\mathbb{L}}(p(\xi, t+s, \cdot), p(\xi, t, \cdot)) \leq \epsilon \quad \forall t \geq T_{1}, s>0
$$

That is,

$$
\sup _{f \in \mathbb{L}}\left|E f\left(X_{t+s}(\xi)\right)-E f\left(X_{t}(\xi)\right)\right| \leq \epsilon
$$

for any $t \geq T_{1}$ and $s>0$. Now, for any $f \in \mathbb{L}$ and $t, s>0$, we compute

$$
\begin{aligned}
\mid E f & \left(X_{t+s}(\xi)\right)-E f\left(X_{t}(\xi)\right) \mid \\
& =\left|E\left[E\left(f\left(X_{t+s}(\xi)\right) \mid \mathcal{F}_{s}\right)\right]-E f\left(X_{t}(\xi)\right)\right| \\
& =\left|\int_{H} E f\left(X_{t}(\zeta)\right) p(\xi, s, d \zeta)-E f\left(X_{t}(\xi)\right)\right|
\end{aligned}
$$




$$
\begin{aligned}
& \leq \int_{H}\left|E f\left(X_{t}(\zeta)\right)-E f\left(X_{t}(\xi)\right)\right| p(\xi, s, d \zeta) \\
& \leq 2 p\left(\xi, s, \bar{H}_{R}\right)+\int_{H_{R}}\left|E f\left(X_{t}(\zeta)\right)-E f\left(X_{t}(\xi)\right)\right| p(\xi, s, d \zeta)
\end{aligned}
$$

where $H_{R}=\{\xi \in D([-\tau, 0], H):\|\xi\| \leq R\}$ and $\bar{H}_{R}=D([-\tau, 0], H) \backslash H_{R}$. By the argument (13), there exists a positive number $R$ sufficiently large for which

$$
p\left(\xi, s, \bar{H}_{R}\right)<\frac{\epsilon}{4}, \quad \forall s>0 .
$$

On the other hand, by Lemma 4.3, there exists a $T_{3}>0$ such that

$$
\sup _{f \in \mathbb{L}}\left|E f\left(X_{t}(\zeta)\right)-E f\left(X_{t}(\xi)\right)\right| \leq \frac{\epsilon}{2}, \quad t \geq T_{3} .
$$

Substituting (30), (31) into (29) yields

$$
\left|E f\left(X_{t+s}(\xi)\right)-E f\left(X_{t}(\xi)\right)\right| \leq \epsilon, \quad t \geq T_{3}, s>0 .
$$

Since $f$ is arbitrary, the desired inequality (28) must hold.

Based on the results above, we can now state our main result.

Theorem 4.1 Let the assumptions $(\mathrm{H} 1)-(\mathrm{H} 4)$ and $(12)$ hold; then the process $Y(t)=X_{t}$ is stable in the distribution.

Proof By Definition 4.1, it suffices to prove that there is a probability measure $\pi(\cdot) \in$ $\mathcal{P}(D([-\tau, 0], H))$ such that for any $\xi \in D([-\tau, 0], H)$, the transition probabilities $\{p(\xi, t, \cdot)$ : $t \geq 0$ \} converge weakly to $\pi(\cdot)$. According to the well-known fact that the weak convergence of probability measure is a metric concept (see [19]), we therefore need to show that, for any $\xi \in D([-\tau, 0], H)$,

$$
\lim _{t \rightarrow \infty} d_{\mathbb{L}}(p(\xi, t, \cdot), \pi(\cdot))=0 .
$$

By Lemma 4.4, $\{p(\eta, t, \cdot): t \geq 0\}$ is Cauchy in the metric space $\mathcal{P}(D([-\tau, 0], H))$ for any fixed $\eta \in D([-\tau, 0], H)$. Therefore, there exists a probability measure $\pi(\cdot)$ such that

$$
\lim _{t \rightarrow \infty} d_{\mathbb{L}}(p(\eta, t, \cdot), \pi(\cdot))=0 .
$$

Furthermore, for any $\xi \in D([-\tau, 0], H)$, Lemma 4.3 shows that

$$
\lim _{t \rightarrow \infty} d_{\mathbb{L}}(p(\xi, t, \cdot), p(t, \eta, \cdot))=0 .
$$

Hence

$$
\begin{aligned}
\lim _{t \rightarrow \infty} d_{\mathbb{L}}(p(\xi, t, \cdot), \pi(\cdot)) & \leq \lim _{t \rightarrow \infty}\left[d_{\mathbb{L}}(p(\xi, t, \cdot), p(\eta, t, \cdot))+d_{\mathbb{L}}(p(\eta, t, \cdot), \pi(\cdot))\right] \\
& =0
\end{aligned}
$$

as required. 
To demonstrate the applications of Theorem 4.1, we give an illustrative example, motivated by [20, Example 4.1].

Example 4.1 Let $\phi: \mathbb{R} \mapsto \mathbb{R}$ be Lipschitzian, i.e., there exists $L>0$ such that $|\phi(x)-\phi(y)| \leq$ $L|x-y|, x, y \in \mathbb{R}$. Assume further that $\varphi:[-\tau, 0] \times[0, \pi] \times[0, \pi] \mapsto \mathbb{R}$ is measurable such that $\varphi(\cdot, \cdot, 0)=\varphi(\cdot, \cdot, \pi)=0$ and

$$
N:=\int_{0}^{\pi} \int_{0}^{\pi}\left(\frac{\partial}{\partial x} \varphi(-\tau, \zeta, x)\right)^{2} d \zeta d x<\infty .
$$

Consider the following stochastic neutral partial functional differential equation:

$$
\begin{aligned}
& d\left[u(t, x)+\int_{0}^{\pi} \varphi(-\tau, \zeta, x) u(t-\tau, \zeta) d \zeta\right] \\
& \quad=\left[\frac{\partial^{2}}{\partial x^{2}} u(t, x)+\phi(u(t-\tau, x))\right] d t+d Z(t, x),
\end{aligned}
$$

with the Dirichlet boundary condition

$$
u(t, 0)=u(t, \pi)=0, \quad t \in[0, T]
$$

and the initial condition

$$
u(\theta, x)=\psi(\theta, x), \quad \theta \in[-\tau, 0], x \in(0, \pi) .
$$

Let $H=L^{2}[0, \pi] ; A$ is given by

$$
\left\{\begin{array}{l}
A:=\frac{\partial^{2}}{\partial x^{2}}, \\
D(A)=H^{2}(0, \pi) \cap H_{0}^{1}(0, \pi),
\end{array}\right.
$$

where $H^{k}(0, \pi), k=1,2$, represent Sobolev spaces, and $H_{0}^{1}(0, \pi)$ is the subspace of $H^{1}(0, \pi)$ of all functions vanishing at 0 and $\pi$. Then we get

$$
A u=\sum_{n=1}^{\infty}-n^{2}\left\langle u, e_{n}\right\rangle_{H} e_{n}
$$

where $e_{n}(x)=\sqrt{\frac{2}{\pi}} \sin n x, n=1,2, \ldots, x \in[0, \pi]$. Let $Z(t, x):=\sum_{n=1}^{\infty} n^{\beta} Z_{n}(t) e_{n}(x)$, where $\alpha \beta \in(0,1)$ and $\left\{Z_{n}(t)\right\}_{n \geq 1}$ is an independent, real-valued, normalized, symmetric $\alpha$-stable process sequence. It is trivial to see that $\delta=\sum_{n=1}^{\infty} \frac{1}{n^{2(1-\alpha \theta)-\alpha \beta}}$. Due to $\alpha \beta \in(0,1)$, we have $1<2-\alpha \beta<2$. Hence there exists $\theta \in(0,1)$ such that $1<2(1-\alpha \theta)-\alpha \beta<2$, and therefore $\delta=\sum_{n=1}^{\infty} \frac{1}{n^{2(1-\alpha \theta)-\alpha \beta}}<\infty$. In other words, the assumption (H4) holds for such a case.

For $t \in[0, T]$ and $x \in[0, \pi]$, let

$$
X(t)(x):=u(t, x), \quad g(X(t-\tau))(x):=\int_{0}^{\pi} \varphi(-\tau, \zeta, x) u(t-\tau, \zeta) d \zeta
$$

and

$$
f(X(t-\tau))(x):=\phi(u(t-\tau, x)) .
$$


Then Eq. (33) can be rewritten in the form (1). Observe that $A$ generates a strongly continuous semigroup $\left\{e^{t A}\right\}_{t \in[0, T]}$, which is compact, analytic and self-adjoint, and

$$
e^{t A} u=\sum_{n=1}^{\infty} e^{-n^{2} t}\left\langle u, e_{n}\right\rangle_{H} e_{n}, \quad u \in H
$$

Thus (H1) holds and $\left\|e^{t A}\right\| \leq e^{-t}$. Furthermore note that

$$
\begin{aligned}
& (-A)^{-\frac{1}{2}} \zeta=\sum_{n=1}^{\infty} \frac{1}{n}\left\langle\zeta, e_{n}\right\rangle e_{n}, \quad \zeta \in H, \\
& (-A)^{\frac{1}{2}} \zeta=\sum_{n=1}^{\infty} n\left\langle\zeta, e_{n}\right\rangle e_{n}, \quad \zeta \in D\left((-A)^{\frac{1}{2}}\right)
\end{aligned}
$$

which in particular yields $\left\|(-A)^{-\frac{1}{2}}\right\|=1$. As a result, using $\varphi(\cdot, \cdot, 0)=\varphi(\cdot, \cdot, \pi)=0$, together with (32), (34), and Hölder's inequality, we get

$$
\begin{aligned}
\|( & -A)^{\frac{1}{2}}(g(X(t-\tau))-g(Y(t-\tau))) \|_{H}^{2} \\
& =\left\|\sum_{n=1}^{\infty} n\left(g(X(t-\tau))-g(Y(t-\tau)), e_{n}\right\rangle_{H} e_{n}\right\|_{H}^{2} \\
& =\sum_{n=1}^{\infty}\left(n \int_{0}^{\pi}(g(X(t-\tau))(x)-g(Y(t-\tau))(x)) e_{n}(x) d x\right)^{2} \\
& =\sum_{n=1}^{\infty}\left(n \int_{0}^{\pi} \int_{0}^{\pi} \varphi(-\tau, \zeta, x) C(t-\tau, \zeta) d \zeta e_{n}(x) d x\right)^{2} \\
& =\sum_{n=1}^{\infty}\left(\int_{0}^{\pi} \int_{0}^{\pi} \frac{\partial}{\partial x} \varphi(-\tau, \zeta, x) C(t-\tau, \zeta) d \zeta \tilde{e}_{n}(x) d x\right)^{2} \\
& =\sum_{n=1}^{\infty}\left(\int_{0}^{\pi} \frac{\partial}{\partial x} \varphi(-\tau, \zeta, \cdot) C(t-\tau, \zeta) d \zeta,\left.\tilde{e}_{n}\right|_{H} ^{2}\right. \\
& =\left\|\int_{0}^{\pi} \frac{\partial}{\partial x} \varphi(-\tau, \zeta, \cdot) C(t-\tau, \zeta) d \zeta\right\|_{H}^{2} \\
& =\int_{0}^{\pi}\left(\int_{0}^{\pi} \frac{\partial}{\partial x} \varphi(-\tau, \zeta, x) C(t-\tau, \zeta) d \zeta\right)^{2} d x \\
& \leq \int_{0}^{\pi} \int_{0}^{\pi}\left(\frac{\partial}{\partial x} \varphi(-\tau, \zeta, x)\right)^{2} d \zeta d x \cdot \int_{0}^{\pi} C^{2}(t-\tau, \zeta) d \zeta \\
& \leq N\|X(t-\tau)-Y(t-\tau)\|_{H}^{2},
\end{aligned}
$$

where $C(t)=X(t)-Y(t)$ and $\tilde{e}_{n}:=\sqrt{\frac{2}{\pi}} \cos n x$, which is also a complete orthonormal system of $H$. Hence, (H3) holds. Consequently, by Theorem 3.1, there exists a unique mild solution to Eq. (33). In addition, Let $p=\frac{3}{2}, k=\frac{1}{2}, \lambda=1, K_{1}=L, K_{2}=\sqrt{N}$; if $10\left(N^{\frac{3}{4}}+M_{\frac{1}{2}}^{\frac{3}{2}} N^{\frac{3}{4}} \mid \Gamma\left(-\frac{1}{2}\right)^{\frac{1}{2}}+L^{\frac{3}{2}}\right)<1$, then by Theorem 4.1, Eq. (33) is stable in the distribution. 


\section{Competing interests}

The authors declare that they have no competing interests.

\section{Authors' contributions}

All authors contributed equally to the manuscript. All authors read and approved the final manuscript.

\section{Acknowledgements}

We are very grateful to the anonymous referees and the associate editor for their careful reading and helpful comments. This work was supported by the National Natural Sciences Foundation of China (No. 11071259, 11371374), Research Fund for the Doctoral Program of Higher Education of China (No. 20110162110060).

Received: 15 September 2013 Accepted: 16 December 2013 Published: 09 Jan 2014

\section{References}

1. Bao, J, Hou, Z: Existence of mild solutions to stochastic neutral partial functional differential equations with non-Lipschitz coefficients. Comput. Math. Appl. 59, 207-214 (2010)

2. Caraballo, T, Real, J, Taniguchi, T: The exponential stability of neutral stochastic delay partial differential equations. Discrete Contin. Dyn. Syst., Ser. A 18, 195-313 (2007)

3. Yuan, C, Mao, X: Asymptotic stability in distribution of stochastic differential equations with Markovian switching. Stoch. Process. Appl. 103, 277-291 (2003)

4. Yuan, C, Zou, J, Mao, X: Stability in distribution of stochastic differential delay equations with Markovian switching Syst. Control Lett. 50, 195-207 (2003)

5. Tan, L, Jin, W, Hou, Z: Weak convergence of functional stochastic differential equations with variable delays. Stat. Probab. Lett. 83, 2592-2599 (2013)

6. Bao, J, Truman, A, Yuan, C: Stability in distribution of mild solutions to stochastic partial differential delay equations with jumps. Proc. R. Soc. Lond., Ser. A, Math. Phys. Eng. Sci. 465, 2111-2134 (2009)

7. Yang, Z, Yin, G: Stability of nonlinear regime switching jump diffusion. Nonlinear Anal. 75, 3854-3873 (2012)

8. Da Prato, G, Zabczyk, J: Stochastic Equations in Infinite Dimensions. Encyclopedia of Mathematics and Its Applications, vol. 45. Cambridge University Press, Cambridge (1992)

9. Dong, Z, Xu, L, Zhang, X: Invariant measures of stochastic 2D Navier-Stokes equation driven by $\alpha$-stable processes. Electron. Commun. Probab. 16, 678-688 (2011)

10. Priola, E, Zabczyk, J: Structural properties of semilinear SPDEs driven by cylindrical stable processes. Probab. Theory Relat. Fields 149, 97-137 (2011)

11. Xu, L: Ergodicity of the stochastic real Ginzburg-Landau equation driven by $\alpha$-stable noises. Stoch. Process. Appl. 123, 3710-3736 (2013)

12. Applebaum, D: Levy Processes and Stochastic Calculus, 2nd edn. Cambridge University Press, Cambridge (2009)

13. Sato, K: Lévy Processes and Infinitely Divisible Distributions. Cambridge University Press, Cambridge (1999)

14. Pazy, A: Semigroups of Linear Operators and Applications to Partial Differential Equations. Springer, New York (1983)

15. Mohammed, S-EA: Stochastic Functional Differential Equations. Pitman, Boston (1984)

16. Bao, J, Hou, Z, Yuan, C: Stability in distribution of neutral stochastic differential delay equations with Markovian switching. Stat. Probab. Lett. 79, 1663-1673 (2009)

17. Long, S, Teng, L, Xu, D: Global attracting set and stability of stochastic neutral partial functional differential equations with impulses. Stat. Probab. Lett. 82, 1699-1709 (2012)

18. Chen, $\mathrm{H}$ : Integral inequality and exponential stability for neutral stochastic partial differential equations with delays. J. Inequal. Appl. 2009, Article ID 297478 (2009)

19. Ikeda, N, Watanable, S: Stochastic Differential Equations and Diffusion Process. North-Holland, Amsterdam (1981)

20. Hernandez, E, Henriquez, HR: Existence results for partial neutral functional differential equations with unbounded delay. J. Math. Anal. Appl. 221, 452-475 (1998)

10.1186/1687-1847-2014-13

Cite this article as: Zang and Li: Stability in distribution of neutral stochastic partial differential delay equations driven by $\alpha$-stable process. Advances in Difference Equations 2014, 2014:13

\section{Submit your manuscript to a SpringerOpen ${ }^{\ominus}$ journal and benefit from:}

- Convenient online submission

Rigorous peer review

- Immediate publication on acceptance

- Open access: articles freely available online

- High visibility within the field

- Retaining the copyright to your article

Submit your next manuscript at $>$ springeropen.com 